\title{
Mythes et rites chez les Anga
}

\section{Pierre Lemonnier}

\section{OpenEdition}

\section{Journals}

\section{Édition électronique}

URL : http://journals.openedition.org/jso/6070

DOI : 10.4000/jso.6070

ISSN : $1760-7256$

\section{Éditeur}

Société des océanistes

\section{Édition imprimée}

Date de publication : 15 décembre 2010

Pagination : 209-220

ISBN : 978-2-85430-027-7

ISSN : 0300-953x

\section{Référence électronique}

Pierre Lemonnier, «Mythes et rites chez les Anga », Journal de la Société des Océanistes [En ligne], 130-131 | 2010, mis en ligne le 15 décembre 2013, consulté le 09 juin 2020. URL : http:// journals.openedition.org/jso/6070; DOI : https://doi.org/10.4000/jso.6070

(C) Tous droits réservés 


\title{
Mythes et rites chez les Anga
}

\author{
par
}

Pierre LEMONNIER*

\section{RÉSUMÉ}

Organisés par les hommes sous l'autorité de maîtres des initiations, les rites masculins des Anga de Papouasie Nouvelle-Guinée mettent classiquement en scène une mise à mort symbolique, puis une renaissance des initiés. Malgré des théâtres végétaux et des gestes globalement semblables, les significations et le détail des actions rituelles varient parallèlement aux mythes d'origine des rituels selon une transformation structurale qu'il est possible de préciser. La comparaison des rites Ankave et Baruya montre des modifications conjointes de leur déroulement, de leur décor et de leur exégèse qui renvoient aux êtres et entités surnaturels auxquels les mythes d'origine de ces rituels attribuent les substances, les pouvoirs et les mises en relations avec des êtres primordiaux qui transforment les jeunes garçons. Pour le sujet qui y participe, ces rituels sont l'occasion de percevoir de façon non verbale quelques-unes des valeurs centrales de la société où il grandit.

MoTS-CLÉS : mythe, rituel, transformation structurale, Anga

Comprendre la part des comportements et pensées individuels dans les phénomènes collectifs constitue l'une de ces quadratures du cercle sur lesquelles buttent les sciences humaines: mettre en rapport les niveaux «micro» et «macro » d'une analyse économique ou historique est aussi délicat que de replacer l'individu dans la société en sociologie. Un degré de complexité supplémentaire est franchi dans cette

\begin{abstract}
Organized by men under the supervision of initiations masters, the male rituals of the Anga of Papua New Guinea classically stage a symbolic death followed by a rebirth of the initiates. The vegetal theatres at hand and the gestures observed look roughly similar, but the detail and meanings attributed to ritual actions vary according to a structural transformation that may be delineated. The comparison of Ankave and Baruya rituals shows concomitant modifications of their procedure, setting, and exegesis which relates to the supernatural beings and entities to which the origin myth of the rituals grant the powers, substances, and relations with primordial beings which transform the young boys. For the individual submitted to them, these rituals are an opportunity for a non verbal perception of some of the key values of the society in which he grows up.
\end{abstract}

KEYwORDS: myth, ritual, structural transformation, Anga

mise en rapport de phénomènes individuels inconscients avec des phénomènes collectifs largement «non intentionnels ${ }^{1}$ » qu'illustre l'anthropologie psychanalytique proposée par Bernard Juillerat dans ses études de «la mort yafar » ou du rite totémique yangis (Juillerat, 1991, 1999). Il s'agissait pour lui, d'une part, de comprendre comment s'effectue «la métamorphose d'images individuelles inconscientes en 61).

* Directeur de recherches au CNRs, Université de Provence, Centre de recherche et de documentation sur l'Océanie (CREDO), Marseille,pierre.lemonnier@univ-provence.fr 
motifs élaborés par l'imaginaire collectif $»$; d'autre part de saisir comment une diversité culturelle « illimité(e) » est produite à partir de ces universaux que sont les «fantasmes originaires » $(2001: 40)$.

En ce domaine, les obstacles sont immenses, qui vont des difficultés pratiques de cette coordination ou «complémentarité » de points de vue que Georges Devereux (1972) avait déjà tentée à la redoutable question des conditions de généralisation des résultats obtenus : pourquoi des institutions sociales pour partie engendrées par des phénomènes psychiques incontestablement universels ne se retrouvent-elles pas partout? « S'il était possible de suggérer une explication satisfaisante [...], d'autres l'auraient fait avant moi », écrivait Juillerat (2004: 160) à propos d'une remarque facile que je m'étais permise à propos de ce mystère agaçant.

Dans le cas d'institutions ayant à voir avec des processus de transformation de la personne, la difficulté de la tâche est d'autant plus irritante que la pertinence d'un rapprochement entre psychanalyse et anthropologie paraît pourtant évidente. Il ne fait aucun doute, par exemple, que les pensées collectivement élaborées et partagées autour de la mort ont un rapport avec le deuil individuel (Lemonnier, 2006 : 171-177). Et une semblable perméabilité est plus que plausible entre l'histoire du sujet participant et les représentations collectives qui, de diverses manières (mythes d'origine, commentaires, injonctions...) accompagnent des cérémonies d'initiation masculine, ne serait-ce que parce que celles-ci impliquent une mort symbolique des garçons et constituent une sortie de l'enfance, une séparation d'avec la mère et, plus largement, un bouleversement relationnel au moment où l'accès à la vie sexuelle est socialement ouvert - sans parler des épreuves psychiques infligées aux novices.

Grande reste notre difficulté à formuler des problématiques situées à l'intersection des approches psychanalytiques et anthropologiques. Par exemple, dans le domaine du rituel, afin de " procéder aux ajustements nécessaires " pour rendre compte de son éventuelle « origine pulsionnelle », ou bien pour saisir en quoi et comment « un rite est un acte symbolique supposé satisfaire le désir que le ou les acteurs y investissent sans le savoir » (Juillerat, $2002: 48$ ). En nourrissant par l'ethnographie la plus fine l'exercice théorique et méthodologique qu'il proposait, Bernard Juillerat n'en a pas moins montré qu'il est possible de faire référence au sujet, voire aux affects et aux conflits du psychisme dans l'étude des rituels. C'était pour lui une façon d'introduire dans l'analyse de ceux-ci le sens des actions en jeu, tant pour celui qui les subit que pour ceux qui les organisent, les regardent, ou les commentent.

C'est en écho à cette exploration par Juillerat de relations plausibles entre divers domaines de la vie sociale et de la vie des individus que j'aborde ici conjointement des variantes des rites d'initiation et des mythes s'y rapportant chez deux groupes anga de Papouasie NouvelleGuinée pour y repérer des variantes de type structural. Pareille démarche ne va pas de soi. D'abord, semblable analyse simultanée des rites et des mythes est illusoire selon Lévi-Strauss, qui parlait alors de la construction d'un « [...] objet hybride dont on peut dire n'importe quoi » (1971 : 598). Quant à l'approche structuraliste, on sait que Bernard Juillerat (par exemple, 2001 : 46) lui reprochait d'impliquer l'effacement du sujet notamment lorsque Lévi-Strauss (1971: 598-601) pose comme préalable de l'analyse du rituel «pur » de séparer celle-ci de l'étude des mythes et des paroles - propos qui rejoint celui du directeur de thèse de Bernard, Roger Bastide (1972 : 207), qui, déjà, regrettait la « disparition progressive de l'affectivité » dans le structuralisme de Lévi-Strauss, tout comme le firent d'ailleurs Beidelman (1966 : 402), Fortes (1969 : 8-9), Leach (1970:8) et Turner (1969: 42-43), auxquels Lévi-Strauss répond dans L'Homme nu (Lévi-Strauss, 1971 : 597).

Mais le terrain est têtu, ce terrain que Bernard a privilégié tout au long de sa pratique de l'anthropologie. En l'occurrence, l'observation de rites masculins ankave et baruya incite à regarder comment ceux-ci et les mythes qui les accompagnent varient de concert. Simplement parce que, derrière d'évidentes ressemblances car, vus de loin, ces rituels paraissent identiques - se profilent des différences qui, à chaque instant, rappellent que l'on se trouve dans des univers à la fois proches et contrastés, condition idéale pour repérer ces "seuils » à partir desquels s'effectue la différenciation culturelle (Juillerat, $2001: 69 s q$ ). Nous verrons que l'analyse comparée des mythes et des rites dit également quelque chose de ces zones d'ombres dont il déplorait qu'elles sont perdues pour l'histoire et relèvent de "facteurs multiples que les sciences sociales sont incapables de maîtriser » (2001 : 72-73).

L'histoire et le structuralisme font mauvais ménage mais il n'empêche que les rites que nous observons aujourd'hui chez les Anga et les mythes que nous y recueillons se sont historiquement modifiés. Certes à la suite d'événements qui nous échappent à jamais et qui furent en leur temps éprouvés et interprétés par des individus 
au gré de cette « créativité, par l'expérience psychique renouvelée (individuelle et collective) vécue par les acteurs »(Juillerat, $2001: 47)$. Mais ces inventions et cette diffusion des mythes et des rites se sont effectuées selon des lignes de fractures et des oppositions que leur analyse comparée révèle, en même temps qu'elle signale ceux de leurs éléments sur lesquels des co-variations ont porté, et même les conséquences de ces mutations pour le contexte culturel dans lequel les individus naissent et se développent. Bref, il est peut-être possible de s'approcher de ces "processus historiques de transmission culturelle [qui] travaillent, avec le temps, à ordonner [les] représentations, à les organiser en récits mythiques, en scénarios rituels, en structure sociale, bref en une ontologie et une cosmogonie qui définiront telle société particulière de façon différentielle» (2001 : 155).

\section{Des comparaisons contrôlées : décrire et commen- ter des variantes locales}

Les Anga des provinces du Gulf, du Morobe et des Eastern Highlands de Papouasie NouvelleGuinée offrent des conditions particulièrement favorables à des études comparatives fines, car il y a toutes les raisons de penser que les groupes linguistiques (et culturels) que l'on rassemble sous ce nom descendent tous d'une protoculture commune qui s'est différenciée sous divers rapports : langues ; types d'économie développés (forme d'horticulture, place du porc semidomestique et des activités de chasse et de pêche); nature et dimension des établissements humains ; formes du mariage (échanges de sœurs au nord, compensation matrimoniale ailleurs) ; importance relative de la guerre; rapports hommes/femmes; coopération entre familles (systématique au nord, évitée à tout prix au sud) (Lemonnier, 1997). Plusieurs des variantes observées dans ces domaines n'ont rien de secondaires, notamment les principes et formes du cycle initiatique.

Celui-ci tient une place centrale ${ }^{2}$ chez les Anga car ces sociétés acéphales organisées en clans patrilinéaires (exogames ou non) se distinguent globalement de celles des autres montagnards de l'intérieur de la Papouasie Nouvelle-Guinée par l'absence d'échanges cérémoniels intergroupes et, donc, de leur organisateur, le Big man (Godelier, 1982 : 253-290 ; Lemonnier, 1990). Dans le monde de « Grands hommes » qui est le leur, les affaires qui mobilisent l'attention collective sont la guerre et les initiations masculines (Godelier, 1982).

Une façon relativement simple d'étudier les variantes des organisations sociales et des systèmes de pensées au sein de l'ensemble culturel anga consiste à comparer deux groupes présentant de forts contrastes socio-culturels. Il importe cependant de souligner à nouveau que ce que les anthropologues observent depuis le milieu des années 1930 chez les Anga sous la forme de cultures et d'organisations sociales distinctes et bien localisées ne représente pas tout l'éventail de ces configurations historiques, mais seulement quelques-uns des résultats de longs processus de transformation historique. Ceux-ci nous échappent car nous ne savons rien des configurations culturelles du passé de cette région de Nouvelle-Guinée qui furent celles de groupes qui ont disparu ou ont été absorbés par d'autres. C'est l'une des raisons pour lesquelles il n'est pas d'actualité de comprendre pourquoi une partie de ces groupes ont, par exemple, produit une variante comme celle qui consiste à " passer » de l'échange des sœurs à un mariage par compensation matrimoniale, ni d'expliquer l'éventuelle ambiguité du statut des oncles maternels ou le choix de principes vitaux masculins plutôt que féminins pour effectuer la « renaissance » des initiés.

En revanche, à défaut d'expliquer les contrastes, c'est-à-dire les variantes que rapporte l'ethnographie des Anga, il est possible de découvrir sur quoi certaines transformations ont porté dans plusieurs domaines se prêtant à cet exercice, comme les rapports entre chamanisme, maladie et guerre (Lemonnier, 1998), le statut de la horde cannibale de morts anciens (Lemonnier, 2006) ou certains aspects des initiations masculines, comme je vais le faire ici.

Les deux sociétés - les Ankave et les Baruya dont je vais comparer les rites masculins et les mythes associés à ces cérémonies occupent des vallées séparées par cinq jours de marche et ne présentent entre elles aucun lien historique connu autre qu'une commune présence, aussi ancienne qu'indatable, dans la haute vallée de Menyamya (province du Morobe), avant leur migration respective vers leur territoire actuel. Il s'agit dans les deux cas d' " horticulteurs » forestiers éleveurs de porcs, mais, situées plus au sud de la cordillère centrale et ouvertes vers les basse terres de Papouasie, les vallées des Ankave sont entièrement couvertes de forêt, alors que celles des Baruya se sont partiellement transformées

2. Une place centrale, et non la place centrale, car plusieurs sociétés anga, dont les Ankave, organisent également d'importantes cérémonies funéraires (Lemonnier, 2006). 
en savane anthropique à force d'y ouvrir des jardins (patate douce, taro, canne à sucre, etc.). Il existe environ dix mille locuteurs baruya, répartis en cinq groupes locaux, dont la « tribu » des Baruya, forte de 3500 personnes (Godelier, 1982). La langue ankave n'est parlée que par 1200 personnes formant une entité politique et territoriale unique. Les territoires des deux groupes sont de superficies équivalentes, de l'ordre de $1000 \mathrm{~km}^{2}$, mais la densité de population des Ankave est quatre fois moindre $\left(1,2 \mathrm{hab} / \mathrm{km}^{2}\right)$. L'habitat ankave est dispersé et fluctuant, ce qui tranche, là encore, avec la situation des Baruya, dont les hameaux permanents sont toujours remplis de monde alors que ceux des Ankave sont généralement déserts (Bonnemère et Lemonnier, 2007 : 57-88).

Au-delà de ces différences patentes observables dans le paysage, la résidence ou l'organisation des jardins (collectifs chez les Baruya, strictement individuels chez les Ankave), les Ankave se distinguent aussi des Baruya en ne pratiquant qu'incidemment l'échange des sœurs (dont l'idée évoque pour les Ankave un mariage « de chiens et de porcs ", mais certainement pas d'êtres humains!), par leur refus quasi explicite de la coopération, mais également par la place qu'ils font aux rites de mort, par la douceur relative de la domination masculine, leur souci des monstres cannibales et leur ignorance totale de l'homosexualité ritualisée qui, par le passé, caractérisait les initiations des Anga du Nord, Baruya, Sambia, Iqwaye (Godelier, 1982; Herdt, 1981, 1987 ; Mimica, 1981, 1991).

\section{Femmes, guerriers et initiés}

Avec leurs langues et leurs cultures matérielles, le complexe de relations que les Anga ont développé entre la guerre, les rapports hommes/femmes et les initiations masculines sont l'une des originalités qui distinguent cet ensemble culturel parmi les populations de Mélanésie. Au cœur de ce système d'institutions et de relations sociales se trouve présente, chez tous les Anga - bien qu'à des degrés variables -, l'idée que la physiologie sexuelle des femmes exerce un effet néfaste sur les hommes, qu'elle fragilise leur ardeur guerrière et, partant, menace la survie du groupe. Les initiations procurent aux hommes leur force physique et morale, mais c'est aussi dans ce cadre que leur sont transmises les connaissances pratiques qui leur permettent de pallier ce dangereux état de choses.

Ces rites masculins ont pour but explicite de transformer les garçons en hommes adultes, forts et sans peur. Simultanément, ces rituels fondent et réaffirment régulièrement la domination masculine. Les personnages directement impliqués comme responsables dans les initiations ou la guerre sont ceux qui, par leur naissance au sein d'un lignage particulier (maîtres des initiations) ou par leurs talents personnels (grands guerriers et chamanes), s'élèvent au-dessus des autres hommes. D'un groupe linguistique anga à un autre, ce schéma prend des formes différentes constituant autant de variantes, mais les relations entre ordre politique, rapports hommes/femmes, guerre et initiations sont partout celles décrites à l'instant.

Pour les Anga, ces rites masculins trouvent leur origine dans un passé primordial dont parlent les mythes. Ceux-ci rapportent que le premier homme apparu dans notre monde, vers Menyamya, octroya à ceux qui émergeaient derrière lui, ancêtres respectifs des différents groupes linguistiques, leur langue et leurs parures corporelles, avant que chacun entame la pérégrination qui le conduisit sur son territoire actuel. Certains racontent également que les os de l'un de ces hommes des premiers temps furent partagés entre tous les groupes et qu'ils ont servi à fabriquer les poinçons avec lesquels on perfore le septum des garçons initiés. Cette " histoire " de «personne distribuée " (Strathern 1988) est donc à la fois ce qui rapproche un membre d'une entité locale quelconque des autres Anga, et ce qui, par l'utilisation ou le partage d'objets ou de substances qui remontent au temps du mythe, établit un lien entre les hommes, à la fois diachroniquement (depuis que les Anga sont apparus sur la terre et ont appris comment transformer des garçons en hommes adultes) et synchroniquement (entre tous les initiés, dans un groupe donné).

Tant l'homogénéité relative des mythes et des rites d'initiation que leur variabilité ne sont donc pas que des hypothèses d'anthropologues : elles se situent au cœur même du dispositif imaginaire et matériel par lequel les Anga pensent tout à la fois leur humanité propre et une partie de leurs différences et, en tout premier lieu, leurs manières d'initier les garçons, qui constituent un ensemble de pratiques dont chaque groupe anga garde secrètement les particularités, même si la structure générale des rituels masculins est partout semblable.

Pour l'ethnologue, ces rituels, obligatoires pour tous les garçons (9-11 ans) prennent, dans tous les cas et classiquement, la forme d'une mort symbolique puis d'une renaissance dont la miseen scène rituelle rappelle un accouchement, mais 
en essayant de se passer des femmes ${ }^{3}$. Cette seconde étape prend fort logiquement en compte les représentations locales de la vie et de la fabrication d'un enfant (Bonnemère, 2001). En particulier, selon l'humeur corporelle à laquelle on attribue localement la vie des êtres humains, c'est par des pratiques ou à l'aide de produits faisant tantôt référence au sang, tantôt au sperme, que les hommes organisent entre eux la gestation ou la renaissance rituelles de ces nouveaux-nés que sont les initiés.

\section{Deux imaginaires des rites masculins}

Les Baruya considèrent que le Soleil est numwe, le père. Le bandeau rouge ypmoulié dont les hommes se ceignent le front est la « route de feu qui unit les ancêtres des Baruya [...] au Soleil » (Godelier, 1982 : 109-127 ; 1996 : 158, 169), et chaque stade des initiations est l'occasion de prières sifflées au Soleil. Mais surtout, celui-ci et son frère cadet la Lune ont donné aux hommes les objets sacrés (kwaimatñe) détenus par les maîtres des rituels masculins - y compris les os d'aigle pointus utilisés comme poinçons -, ceux au contact desquels les novices reçoivent dans leur corps un peu des «puissances surhumaines qui gouvernent l'univers ", et notamment de la force du Soleil (Godelier 1996 : 169, 173-174). En même temps qu'il offrait ces objets aux hommes, le Soleil a institué les initiations elles-mêmes, expliquant les tâches à faire, y compris les opérations de divination qui permettent aux spécialistes des rituels de repérer les futurs grands hommes.

Les hommes doivent leur sperme au soleil qui, lui-même, intervient dans la conception d'un enfant en donnant la forme finale de l'embryon (Godelier, 1982 : 109). Selon les conceptions locales de la vie, chez les Baruya, la mère n'est que le réceptacle porteur du fœtus. C'est le sperme du père qui construit et fait croître le fotus, ce qui implique des rapports sexuels pendant la grossesse ; le lait de la mère est du sperme du père transformé, celui qu'elle a ingéré par fellation (Godelier, 1982 : 91, 116) ; la canne à sucre produit du sperme chez les hommes et du lait chez les femmes. Cette omniprésence du sperme se retrouve dans les initiations car les novices boivent (buvaient) pendant plusieurs années la semence de leur aînés, ce qui les faisait grandir, tout en remplissant leur propre sac du sperme qu'eux-mêmes feront circuler plus tard entre les sexes et les générations.
La situation est radicalement différente pour les Ankave, qui considèrent que seule la mère fabrique le bébé, à partir d'un mélange de sperme et de sang dont sort l'ébauche du fœus ; l'enfant croît in utero uniquement en partageant le sang de sa mère, dont il se nourrit. Le père n'a rien à voir dans cette fabrication d'un être humain et les rapports sexuels cessent aussitôt la grossesse repérée. Il est alors peu surprenant que, pour ceux qui partagent cette vision de la transmission de la vie, ce processus de renaissance des jeunes garçons qu'est l'initiation masculine mette en jeu le sang, une substance localement considérée comme exclusivement féminine. Les Ankave font donc très logiquement renaître et grandir les novices à travers l'ingestion de jus de pandanus, un substitut du sang du premier homme (que l'on peut qualifier de déféminisé puisque, par définition, celui-ci n'avait pas de mère !) et en les enduisant de cette sauce vermillon (Bonnemère, 1994). Du soleil, il n'est pas question.

Cependant, divers appareils du rituel des Ankave, et notamment des éléments du théâtre végétal qui leur sert de décor à plusieurs reprises, pourraient conduire à des interprétations donnant au soleil un rôle important dans les initiations de ce groupe, alors même qu'il ne fait pas grand-chose dans ces histoires. C'est de cette ambiguïté qu'il importe d'exposer et de commenter les termes. Observer en train de se faire des rites d'apparence semblable et pourtant si différents permet de saisir en quoi, sinon comment, ont parallèlement évolué des imaginaires, des objets, des gestes, et des théâtres rituels qui font plutôt privilégier le sang féminin au sperme dans des représentations de la vie qui retentissent profondément dans la vie des gens.

\section{Quand le Soleil s'éclipse : objets sacrés et rites ankave vus en clef baruya}

À l'instar des Baruya, les Ankave font de l'origine de l'humanité actuelle et de celle des objets sacrés et des initiations deux événements contemporains et liés entre eux qu'ils situent dans la vallée de Menyamya, en un lieu où les mythes ankave rapportent que le premier homme distribua et les langues et les décorations corporelles de tous les groupes linguisticoculturels anga. Incapable de dire son propre nom, l'homme fut mis à mort et, là où se répandit son sang poussèrent le pandanus rouge et les cordylines oremere'. Son esprit expliqua en

3. La mort des novices n'en est évidemment pas une, mais seulement un processus de transformation du corps si dangereux - perforation du septum nasal avec de réels risques de septicémie - qu'il implique un voisinage réel avec la mort. 
rêve aux ancêtres des Ankave avec quoi et comment initier les garçons. Il ordonna de fabriquer un poinçon avec l'un de ses os et de l'utiliser pour perforer le septum des novices, puis de les frotter de jus de pandanus rouge.

Pour l'anthropologue, un objet sacré oremere' (du même nom que les cordylines rouges) appartient clairement à la même classe d'objets que les kwaimatñe des Baruya, avec une immense différence, cependant : l'absence de toute référence au Soleil dans les mythes d'origine des objets sacrés et des initiations elles-mêmes. La place qu'occupe le Soleil chez les Baruya (et d'autres Anga du nord) comme origine des pouvoirs masculins est tenue chez les Ankave par l'homme primordial apparu près de Menyamya. Pour eux, aucune force émanant du Soleil n'est transmise aux initiés, mais seulement celle de ce premier homme, doublée par celle d'un héros légendaire " récent », Natemewo. L'objet oremere' est un être animé et doué de perception puisqu'il sait que des initiations vont avoir lieu dès que le maître des rituels le sort de sa cachette («sonwëwuñe!», « regarde!»). Et c'est à lui qu'il parle lorsqu'il referme et range le paquet magique: "Tu as tué des hommes / enfants; retourne dormir dans ta maison! ». La masculinité ingérée par les futurs guerriers ankave provient du sang du premier homme et non du Soleil dont les Baruya transmettent la force aux novices, tant via le sperme des aînés que par leur contact physique avec le kwaimatñe. Bref, ce n'est pas le Soleil, mais un homme - un représentant de l'humanité actuelle -, sorte de premier ancêtre sans descendance de tous les Anga susceptible d'être partagé et distribué entre eux tous sous forme de reliques humaines, qui est l'acteur principal des cérémonies ${ }^{4}$.

Il suffit pourtant de regarder quelques instants les rites ankave avec les yeux d'un anthropologue spécialiste de leurs lointains cousins baruya pour être, sinon ébloui, au moins interloqué par toute une série d'éléments du décor, par des gestes, et même des paroles, qui prouvent que le Soleil n'est pas loin. Mais pas loin pour qui ? Seulement pour les ethnologues comparatistes à l'affût de variantes, ou également pour les Ankave? Et avec quelles conséquences pour notre connaissance des mécanismes de l'invention et de la transformation des codes culturels ? C'est toute la question.

\section{Un mythe pour éclairer la variante sans Soleil}

Il est deux moments du cycle initiatique ankave où l'impression de " déjà vu » est si forte pour un observateur imprégné de culture baruya qu'il serait très facile de voir le soleil s'y infiltrer. À la fin de la période de réclusion du premier stade, d'abord, les novices sont brutalement propulsés à près d'un mètre en l'air le long d'un immense tronc d'arbre (un Syzygium) que raclent leur ventre et leurs bras. Un spécialiste des Baruya ou des Sambia serait tenté de voir là une transfusion vers le bas-ventre des garçons d'une force descendant du ciel (et du soleil) via le tronc rouge orangé d'un géant de la forêt. Il ne manquerait pas de remarquer que l'on construit parfois ${ }^{5}$, appuyée à l'arbre, une grande plate forme semblable à celle sur laquelle les Baruya hissent un à un les novices muka du premier stade des rituels (Dunlop, 1992 : 35). Pourtant, des heures et des semaines d'enquêtes réparties sur plus de vingt ans auprès d'une quinzaine d'hommes ne m'ont rapporté sur ce point qu'une seule information, toujours la même: "Nous faisons ça aux gamins pour qu'ils n'aient pas peur de grimper aux arbres lorsqu'ils cueillent des noix d'arec ou chassent les marsupiaux ». Puisqu'on me le dit!

Ensuite, lors des rites du troisième stade, appelé tsuwangain, du nom de la décoration d'incisives de cochon remise ce jour-là à un ou deux jeunes hommes dont la femme vient de mettre au monde leur premier enfant, c'est tout le décor végétal du rituel qui impose dans l'esprit de l'observateur l'idée d'une présence du Soleil. Les rhombes hurlent tandis que les participants, tous déjà pères et initiés tsuwangain, arrivent sur les lieux, au moment où le maître des rituels achève de transfigurer un anodin coin de forêt en site sacré. Elle aussi adossée à un énorme arbre à l'écorce rouge (un saoxe', Elaeocarpus sp.), une nappe d'écorce teintée en orange par du jus de pandanus (ogidze) sert de présentoir à toutes

4. «Reliques » est sans doute ici un mot-clef car, dans le cours même des rituels, alors que les Baruya ou les Sambia font passer entre les générations d'initiés du sperme venu d'un père suprême qui est le soleil, c'est à un humain en chair, en sang et en os que les Ankave doivent ce qu'ils sont et ce qui leur permet de se reproduire comme ils sont, c'est-à-dire semblables aux autres Anga et en même temps formidablement différents d'eux. Les pouvoirs que les Ankave transmettent aux initiés sont rendus visibles et efficaces par des objets (os, cordylines gorgées de sang) qui sont, pour eux, d'une même nature - humaine-que les héros dont ils proviennent. J'essaie ailleurs, mais encore sans succès, de démêler toutes les implications de cette mise en reliques (Lemonnier, sd).

5. En 1994, lors des rites du premier stade, une telle plateforme fut construite au pied d'un saoxe', puis immédiatement détruite. On avait sans doute jugé inutile de l'utiliser car les racines aériennes (contreforts) de l'arbre étaient de petite taille, si bien qu'il était possible d'atteindre la partie régulière du tronc depuis le sol. 
sortes de décorations corporelles. Quinze ou vingt de ces arbres, de petit diamètre, sont décorés de feuilles des mêmes saoxe' ramassées sur le sol et qui forment comme des couronnes rouges vif " comme le sang », me dit-on, à deux mètres du sol (Lemonnier, 2005).

La base de ce petit autel repose sur le sol au centre d'un demi-cercle de cordylines rouge pourpre. De type oremere' (le même nom que l'objet sacré), elles sont supposées avoir initialement poussé dans une terre gorgée du sang du héros primordial et l'expert rituel les a coupées dans son propre enclos domestique, puis apportées là en grand secret et disposées sur plusieurs rangs. Des parures de plumes, des colliers de dents de cochons, des barettes nasales, des baudriers et bandeaux de cauris sont posés sur la nappe d'écorce, à peu près à la place qu'ils occuperaient sur un homme. N'était le grand nombre de parures exposées, on devinerait un personnage à demi allongé les pieds dans des cordylines. D'autant que, secoué par un comparse dissimulé sous la nappe ou par l'intermédiaire d'une liane tirée à distance, le grand rectangle orangé tremble régulièrement au rythme d'un homme qui respire (ou expire ?). Le dos appuyé aux petits saoxe' les hommes menacent le novice auquel deux parrains viennent de faire dévaler la pente menant à l'autel. Terrorisé par les haches et les gourdins, le jeune père est enjoint de se placer au milieu des cordylines et de regarder le petit autel sous une grêle de paroles telles que : « Si on dit que les ennemis arrivent, tu vas les regarder et te sauver?».

Après de longues minutes de sermon, de cris violents et de menaces, le novice est amené sous la nappe d'écorce où se cache le maître des initiations, accroupi à côté d'un petit feu qui ne dégage aucune fumée. Lentement, on lui passe les mains et les articulations des bras dans les flammes, si bien que j'ai longtemps eu du mal à ne pas imaginer quelque plomberie cosmique mettant les hommes, les végétaux et les objets présents au pied du saoxe' en connexion avec la puissance du soleil. Mais les Ankave ne sont pas les Baruya. Pour l'expert rituel ankave, ce n'est pas des pouvoirs du soleil qu'il est question, mais de terre suwayé dont la version d'un récit semimythique dit qu'elle contient du sang de Natemowo, ce super guerrier dont la mort dramatique fait un doublon récent de l'ancêtre primordial. Pour l'anthropologue, il ne fait pas de doute que l'être " distribué » allongé devant les novices n'est autre que Natemowo, évoqué sous la forme d'une nappe de ogidze décorée comme celles sur lesquelles on exposait naguère la dépouille des grands combattants. Les cordylines qui l'entourent sont celles qui furent jadis plantées autour du cadavre de ce demi-dieu et qu'on se partagea comme on le fit jadis, selon le mythe, de celles qui poussèrent dans un terre imbibée du sang de l'ancêtre primordial, vers Menyamya.

Le maître des rites tsuwangain me parle aussi des cordylines qu'il déterre et rassemble en évoquant l'arc-en-ciel qui apparaît et se dissipe aussi vite que la transformation des novices est intervenue; et du soleil qui monte, rouge, lorsque l'arc-en-ciel disparaît. Dans cette affaire, le soleil n'est donc pas absent, mais il est comme repoussé à la marge du système imaginaire qui accompagne et rend plausibles les actions magiques (pour nous) qui parachèvent les hommes. À la réflexion, la mise en scène du rituel tsuwangain fait en effet immanquablement penser au mythe ankave d'origine du soleil, car, selon ce récit, c'est le long d'un arbre saoxe' que s'enfuit le marsupial aux yeux «comme le soleil» qu'un chasseur avait blessé en plein foie d'une flèche multipointes. Le sang du marsupial et l'animal lui-même se transformèrent en soleil, et ce sont les pointes de la flèche que l'on voit chaque jour, lorsque les rayons de l'astre passent au-dessus des montagnes, à l'aube. Le sang du marsupial monté au ciel est devenu le soleil rouge du matin. Celui qui s'est répandu au sol a imbibé la terre suwayé - comme le fit bien plus tard celui de Natemowo. Quant aux rayons orangés qui filtrent à travers les nuages, ce sont les gouttes qui s'écoulaient du corps de l'animal pendant son ascension au ciel.

\section{Structure, variantes, scories}

Il est bien question de soleil en filigrane des rites tsuwangain des Ankave, mais c'est d'un marsupial qui monte au ciel que parle leur mythe et, jamais, d'une descente des pouvoirs du soleil. Et c'est parce qu'un humain, un chasseur, a fléché le marsupial-soleil que celui-ci est à sa place dans le ciel. Le Soleil qui vient aider les Baruya lors des initiations et de chaque conception n'existe pas dans l'univers ankave. Ici, les héros fondateurs sont des hommes du passé dont les pouvoirs sont toujours présents dans ces pandanus qui teintent les ogidze $e^{6}$ et dans ces éternelles cordylines oremere' que l'on coupe et replante génération après génération, chaque fois qu'une cérémonie nécessite une enceinte sacrée. C'est

6. Par contraste, les ypmoulié baruya, dont on vu qu'il sont la « route de feu » venant du soleil, sont découpés dans une nappe d'écorce battue préalablement trempée dans du jus de bétel (pour acquérir sa teinte rouge). 
d'ailleurs des actes des humains que parlent les Ankave. À mes insinuations sur le rôle de ce soleil bien rouge dont il évoquait la montée au ciel, le maître des tsuwangain a répondu de la manière la plus claire : "C'est moi seul qui fais ça!». Le soleil, et puis quoi encore! Le soleil n'est pourtant pas loin de l'autel forestier, mais pour qui ? De même, c'est à un homme, le premier, tué par des hommes, de surcroît, que les Ankave attribuent l'origine ambiguë de leurs

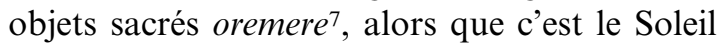
qui a envoyé les kwaimatñe aux Baruya.

Pour les anthropologues, le soleil qui se trouve à la marge des rites ankave est un élément du système rituel masculin dont la signification diffère radicalement de ce qu'elle est chez d'autres groupes anga, dont les Baruya. Et de cette transformation, de cet engendrement de variante, un commentaire $\mathrm{du}$ mythe d'origine $\mathrm{du}$ soleil signale clairement le nœud puisque « le sang du marsupial est devenu le soleil rouge du matin ».

\section{Ce que dit la variante ankave : du sang et du sens}

Appliquée à des matériaux comparables - ici, un même ensemble mythico-rituel de NouvelleGuinée-l'analyse structurale permet de retracer un cheminement logique ou, au moins, de signaler les carrefours de sens, les points de différenciation, les tensions autour desquels un thème mythique et un scénario rituel se réorganisent et prennent une saveur culturelle locale. Dans le cas de sociétés "voisines " au moins, comme c'est le cas des Ankave et des Baruya, ces lignes de clivage à partir desquelles ont été élaborées des variantes mythiques et rituelles à la fois proches et radicalement dissemblables, prennent la forme d'oppositions simples du type de celles mises au jour par le structuralisme lévistraussien.

En comparant des rituels comparables en même temps que les mythes apparentés qu'on leur associe localement, on voit s'affiner les déformations d'un schème culturel plus ou moins général - l'initiation comme séparation d'avec la mère, renaissance entre les mains des hommes et transformation de relations (Bonnemère 2008) - lorsqu'il passe à travers le double filtre d'un ensemble culturel particulier et d'une histoire locale. Cette comparaison au plus près fait ressortir l'accent que les Ankave mettent sur le sang : sang du héros primordial, d'un guerrier formidable, d'un marsupial qui donna naissance au soleil. Contenu dans des argiles, des cordylines ou dans le jus du pandanus rouge, le sang de ces êtres surnaturels est présent lors de ces événements récurrents que sont les rituels. Il ne s'agit pas de manifestations métaphoriques du liquide auquel les Ankave attribuent la vie, mais bien de sa présence réelle. Ne me dit-on pas qu'il suffit de secouer tel entrenœud de bambou pour que l'argile des premiers temps le remplisse à nouveau, aussi humide de sang que lorsqu'un ancêtre l'avait recueillie ? On me fait également remarquer (!) que, sitôt déterrées, les cordylines plantées au pied de l'arbre qui fut le chemin du marsupial-soleil cessent de briller, preuve indirecte de la vie dont elles resplendissent au moment où elles entourent l'autel secret. Quant à l'autel, il vit autant du sang du marsupial grimpé au ciel que de celui de Natemowo - héros qui respire encore, si l'on en juge par les tressaillements de la nappe d'écorce qui le représente.

L'histoire, c'est-à-dire les mythes ou les commentaires des rites que j'observe, ne dit pas toujours si les cordylines rouges utilisées dans les rites masculins ankave sont plutôt emplies du sang de l'homme primordial, de celui de Natemewo ou de celui du marsupial, mais c'est chaque fois de sang qu'il s'agit. Même chose pour l'argile suwayé, que l'on me présente comme tantôt gorgée du sang du premier homme, tantôt de celui du marsupial-soleil. Et c'est encore autour du sang que se joue le tour de passe-passe qui permet aux hommes d'imaginer leur rôle premier - et non celui d'un être du cosmos comme le Soleil des Baruya - dans la machinerie des initiations de deux premiers stades. Les pouvoirs qui y sont mis en jeu sont ceux du sang. Celui-ci est réputé être féminin et maternel par nature, mais c'est bien de sang d'homme que sont gorgés le pandanus, les cordylines et diverses argiles liés à la mort de l'homme primordial car, par définition, ce héros n'a pas de mère.

\section{Et le sujet ?}

L'étude des variantes des mythes et des rites masculins anga révèle des éléments de systèmes de pensée et d'action qui, d'un groupe à l'autre, sont tantôt centraux, tantôt neutres, non marqués, presque transparents. Le fait que les mythes ankave décrivent des arbres rouges semblables à la route à sens unique empruntée par un marsupial blessé pour grimper au ciel et devenir

7. Ambiguë car, si ce sont des hommes qui tuèrent le premier d'entre eux apparu sur la terre, c'est cet homme primordial qui confia en rêve aux Ankave comment initier les garçons avec ses os (poinçon et rhombe) et son sang (présent dans les cordylines et le pandanus rouge). 
le soleil conduit à se souvenir que les Anga du Nord (Baruya et Sambia en l'occurrence) mettent à l'inverse l'accent sur les descentes régulières des pouvoirs de l'astre et leurs interventions dans le monde des hommes. Mais de cette possibilité, les Ankave, eux, ne savent rien et n'ont rien à faire. Tout ou partie des fonctions qui sont celles du Soleil chez les Baruya et les Sambia sont tenues par le sang dans le système de pensée ankave et, par rapport à la constellation mythique des deux premiers groupes, le lien entre les héros (l'homme primordial et Natomewo) et le soleil est comme rompu. Chez les Ankave, la grille d'analyse en clef de soleil ne montre pas autre chose que la trace d'une configuration précédente ou le germe d'une signification autre. Le soleil ressemble fort à un agent secondaire, non marqué, non commenté et non mis en objets. Dans l'hypothèse où les initiations ankave relèvent d'un système de pensée qui fut également commun aux Anga du Nord, le soleil et les bribes de décors rituels qui l'évoquent chez les Ankave se présentent comme des scories en perte de signification d'un système mythique et rituel précédent ou comme des éléments de sens en attente de signification.

Les variantes qui résultent de la transformation conjointe des mythes et des rites que j'ai explorée n'ont guère d'explication; mais elles ont des effets ou des échos quotidiens dans la vie des hommes et des femmes qui les vivent et les mettent en œuvre. On pourrait avancer l'idée que la coopération entre une femme et son époux a peut-être quelque chose de nécessaire dans un groupe comme les Ankave, constitué de familles réfugiées - ou fuyant les monstres cannibales ombo'-, qui exploitent seules, et toujours à quelque distance les unes des autres (Bonnemère et Lemonnier, 2007 : 21-36 ; Lemonnier, 2006 : 39-48), des recoins supposés tranquilles de la forêt. Et qu'il est peut-être alors plus délicat de rendre les femmes totalement responsables des misères des hommes et qu'il devient pensable de leur reconnaître explicitement un rôle dans la fabrication des guerriers comme c'est le cas chez les Ankave?

Au-delà de ces hypothèses presque hasardeuses, mieux vaut considérer qu'il n'y a guère de nécessité dans la logique d'un imaginaire collectif, mais seulement des conventions : pourquoi le sperme et le soleil plutôt que le sang et un héros humanoïde; pourquoi mettre en histoire une création baruya des objets sacrés par le Soleil plutôt qu'une origine ankave du Soleil due à un acte humain (tuer un marsupial) ? En revanche, les éléments de ce système de pensée pananga sont localement cohérents les uns avec les autres. Ainsi, dans des rituels concernant une renaissance, comme c'est le cas des initiations masculines, il est "cohérent» de donner au sperme ou au sang la place qu'ils occupent dans les représentations locales de la conception des êtres humains : l'élément auquel on accorde le rôle le plus important (voire unique) dans la fabrication d'un être humain est celui qui est mis en avant lors de la production des guerriers. Et, là où, pour quelque raison, le sang féminin se voit conférer la part belle dans la croissance d'un embryon, la présence active des mères lors des initiations masculines n'est pas pour surprendre (Bonnemère, 2008).

L'analyse comparée n'éclaire pas la présence d'un ensemble mythe-rite particulier en un lieu et temps donnés, mais, pris tous ensemble, les gestes, les paroles, et les objets du rituel sont autant d'éléments qui se renvoient les uns aux autres et construisent, pour le sujet, l'ambiance culturelle dans laquelle il vit. L'analyse conjointe des mythes et des rites montre sur quoi portent ces transformations, mais aussi, et c'est essentiel, de quelles caractéristiques des organisations sociales ou des représentations ces variations sont concomitantes. Dans le cas des Ankave, tant les mythes que les rites disent, avec et sans mots, l'importance du sang maternel. Mais ce n'est pas tout.

Aux différences que l'anthropologue met au jour, à la fois dans l'imaginaire des initiations, dans les actions rituelles, dans les objets sacrés et dans d'autres éléments matériels du système rituel correspondent des manières de vivre dissemblables, dont les conséquences sociales et individuelles sont lourdes pour les hommes et femmes qui sont membres des groupes en question. Comme le rappelait Juillerat, des rites d'initiation « mettent en scène des sujets réels, individuels (les initiés) et collectifs (des classes d'âge, la communauté masculine), inscrits dans l'histoire du groupe» (Juillerat, 2001 : 264-265). Pour celui qui y participe d'une manière ou d'une autre, c'est-à-dire comme novice, parrain initiatique, organisateur, spectateur, parent d'un initié etc., ce qui se joue dans le cadre rituel est en résonance avec ce qu'il ou elle sait déjà des relations entre les hommes et les femmes, de leurs substances respectives, des substituts de celles-ci et de leurs pouvoirs.

Au-delà des initiations proprement dites, les relations que ces rituels imposent ou non entre un novice (puis un initié puis un jeune homme) et ses parents varient d'une manière qui ne peut que retentir profondément sur la vie intime des sujets, en particulier les modalités de la séparation des garçons d'avec leur mère. Formidablement 
violente et longue de plusieurs années chez les Baruya (Godelier, 1982: 63) et les Sambia (Herdt, 1981 : 135, 216, 289-290), celle-ci n'est que temporaire pour les initiés ankave, qui, dès le second stade, peuvent revenir chez leurs parents après deux mois passés dans une maison de célibataires. Tout aussi sidérantes pour un spécialiste des Anga du Nord sont les actions des femmes durant les rites ankave, actions que les hommes eux-mêmes jugent nécessaires à la réussite de ceux-ci, alors que cette simple idée serait quasi blasphématoire pour un Baruya. De même, on imagine l'importance, là où elles existent, de pratiques susceptibles de retentir sur la structuration psychique des individus : l'homosexualité ritualisée, centrale chez les Baruya et Sambia et inexistante dans les cérémonies masculines des Ankave.

Une co-variation n'est pas la démonstration d'une causalité, mais force est de constater que la reconnaissance par les hommes ankave, sinon de l'origine féminine de la substance à laquelle ils donnent le premier rôle dans les initiations $\left.\right|^{8}$, le sang, au moins du rôle des femmes dans toute naissance, va de pair avec leur part cruciale dans les rites masculins et avec le statut de celles-ci, plutôt enviable si on le compare à celui d'autres femmes Anga, et, d'abord, avec la moindre violence des hommes envers elles (Bonnemère, 1996: 381-382). Car, pour les Ankave, les femmes sont seulement responsables, mais pas coupables des faiblesses des hommes, alors que toutes les misères des hommes sont de leur faute, pour les Baruya ou les Sambia (Godelier, 1982 : 84-90 ; 1996 : 46-51 ; Herdt, 1981 : 225226).

Conséquence directe de cette vision des choses, il n'existe pas d'initiations féminines chez les Ankave (Bonnemère, $1996: 381$ ), alors que, chez les Baruya, où l'on observe une « dénégation de l'importance des femmes dans le procès de reproduction de la vie » (Godelier, 1982: 229), les femmes elles aussi sont initiées, lors de cérémonies où d'autres femmes imposent « à des jeunes filles prêtes parfois à se rebeller contre les règles d'un jeu qui les donnent à un homme dont elles ne veulent pas, l'ordre de leur société qui est l'ordre de la domination masculine » (Godelier, 1982 : 87-89). Rien de tel, donc, n'existe chez les Ankave où la reconnaissance du rôle du sang et des femmes, dans la reproduction de la vie et dans les initiations, est autant lisible et ressentie par le sujet lors des initiations qu'à travers les interactions quotidiennes entre les hommes et les femmes qu'il observe et auxquelles il participe.

À défaut d'explication rendant compte de la production d'un ensemble mythe-rite particulier, la façon dont les mythes et les initiations se transforment et confèrent au sang les pouvoirs qui sont les siens chez les Ankave retentit donc bien au-delà des seuls rituels, puisque, à la cohérence des rapports entre mythe et rite, correspond autant la part du sang et des femmes dans l'action rituelle que le respect relatif dont elles jouissent dans la vie de chaque jour. Lors des initiations, le sujet masculin apprend progressivement des mythes et des significations qui, de diverses manières, débordent de beaucoup le moment rituel proprement dit. Il ressent notamment, dans son corps et dans son esprit, de façon verbale et non verbale, des actions, des émotions et des savoirs relatifs au monde où il est né et où il vit, et qui président aux transformations que sa personne subit lors des cérémonies. Manière de répondre partiellement à la question que se posait Juillerat à propos de l'élaboration et de l'acquisition de « représentations déjà instituées dans la culture (qui) font partie de l'héritage que tout nouveau sujet reçoit au cours de sa socialisation » (2001: 165).

\section{BIBLIOGRAPHIE}

BASTIDE Roger, 1972. Sociologie et psychanalyse, Paris, PUF.

BeIdelman Thomas, 1966. Swazi Royal Ritual, Africa 36 (4), pp. 373-405.

Bonnemère Pascale, 1994. Le pandanus rouge dans tous ses états. L'univers social et symbolique d'un arbre fruitier chez les Ankave-Anga (PapouasieNouvelle-Guinée), Annales Fyssen 9, pp. 21-32.

_, 1996. Le pandanus rouge: Corps, différence des sexes et parenté chez les Ankave-Anga (PNG), Paris, CNRs Éditions/Éditions de la maison des sciences de l'homme, Chemins de l'ethnologie.

_, 2001. Two forms of masculine ritualized rebirth. The Melanesian body and the Amazonian cosmos, in T. Gregor and Don Tuzin (eds), Gender in Amazonia and Melanesia: an Exploration of the Comparative Method, Berkeley, The University of California Press, pp. 17-44.

—, 2008. Du corps au lien : l'implication des mères dans les initiations masculines des Ankave-Anga, in

8. Comme me le fait remarquer Maurice Godelier, le sang du premier homme, présent dans le jus de pandanus rouge qui fait croître les novices avant leur renaissance à la fin du deuxième stade des rituels ne présente aucune ambiguïté car ce héros n'a pas de mère. Celui de Natomowo, en revanche, héros humain « récent », est d'origine indubitablement maternelle. Ce sont alors les cordylines, imbibées du sang des deux héros, qui sont d'origine ambiguë. 
I. Théry et P. Bonnemère (eds), Ce que le genre fait aux personnes, Paris, Éditions de l'EHESs, pp. 75-90.

Bonnemère Pascale et Pierre Lemonnier, 2007. Les tambours de l'oubli. La vie ordinaire et cérémonielle d'un peuple forestier de Papouasie, Papeete, Au Vent des Îles.

Devereux Georges, 1972. Ethnopsychanalyse complémentariste, Paris, Flammarion.

Dunlop Ian, 1992. Baruya Muka Archival. Vol. 2 : Film parts One to Four, Lindfield, Film Australia Pty.

ForTEs Meyer, 1967. Totem and Taboo, Proceedings of the Royal Anthropological Institute... for 1966, London, Royal Anthropological Institute.

GODELIER Maurice, 1982. La production des grands hommes. Pouvoir et domination masculine chez les Baruya de Nouvelle-Guinée, Paris, Fayard.

_, 1996. L'énigme du don, Paris, Fayard.

HerdT, Gilbert, 1981. Guardians of the Flutes. Idioms of masculinity, Chicago and London, University of Chicago Press.

_, 1987. The Sambia: Ritual and Gender in New Guinea, New York, Holt, Rinehart and Winston.

JuILlerat Bernard, 1991. Edipe chasseur. Une mythologie du sujet en Nouvelle-Guinée, Paris, PUF, Le fil rouge.

—, 1999. La mort yafar, L'Homme 149, pp. 63-82.

_, 2001. Penser l'imaginaire. Essais d'anthropologie psychanalytique, Lausanne, Payot Lausanne.

_ 2002. Le rite comme acte et comme représentation : de l'inconscient à l'espace représentationnel, in C. Botella (éd.), Penser les limites. Écrits en l'honneur d'André Green, Paris, Delachaux et Niestlé, pp. 44-51.

—, 2004. Psyche, culture and society, Journal of Ritual Studies 18 (1), pp. 158-166.

LeACH Edmund, 1970. Lévi-Strauss, London, Fontana.
LEMONNIER Pierre, 1990. Guerres et festins. Paix, échanges et compétition dans les Hautes Terres de Nouvelle-Guinée, Paris, Éditions de la maison des sciences de l'homme.

_, 1997. Mipela wan bilas. Identité et variabilité socio-culturelle chez les Anga de Nouvelle-Guinée, in S. Tcherkézoff et F. Marsaudon (éds), Le Pacifique-Sud aujourd'hui : Identités et transformations culturelles, Paris : CNRs Éditions, pp. 196-227.

_ 1998. Maladie, cannibalisme et sorcellerie chez les Anga de Papouasie Nouvelle-Guinée, in M. Godelier et M. Panoff (éds), Le corps humain. Supplicié, possédé, cannibalisé. Paris, Éditions des archives contemporaines, pp. 7-28.

_ 2005. Objets sacrés sans « style ». Circulez, y’a rien à voir !, in B. Martinelli (éd.), Style et fonction stylistique, Marseille, Presses de l'Université de Provence, pp. 237-254.

—, 2006. Le sabbat des lucioles. Sorcellerie, chamanisme et imaginaire cannibale en Nouvelle-Guinée Paris, Stock.

—, s.d. Quand la monnaie n'est pas une personne, les morts ne sont pas des ancêtres. Variations sur la partition des êtres en Mélanésie, manuscrit non publié.

LÉVI-STrauss, Claude, 1971. L'homme nu, Paris, Plon.

MimicA Jadran, 1981. Omalyce. An ethnography of the Ikwaye view of cosmos, Doctoral dissertation, Canberra, Australian National University.

_, 1991. The incest passions: An outline of the logic of the Iqwaye social organization, Part 2, Oceania 62, pp. 81-113.

STRATHERN Marilyn, 1988. The Gender of the Gift. Problems with Women and Problems with Society in Melanesia, Berkeley, Los Angeles, London, University of California Press.

Turner Victor, 1969. The Ritual Process. Structure and Anti-Structure, Chicago, Aldine Publishing Company. 
